\title{
Audiological evaluation in children and adolescents with turner syndrome by otoacustic emissions
}

\begin{abstract}
Turner syndrome (TS) is characterized by short stature and hypergonadotrophic hypogonadism, associated with typical somatic features. Although hearing loss (HL) was not reported in Henry Turner first description of the disease, it has been brought to attention in the last decades in many studies. But the true nature of this problem is still unknown due to inconsistent data resulting from different study methodology. The purpose of this study is to evaluate hearing of TS girls using Audiometry and Otoacustic emissions, both Distortion product (DPOAEs) and Transient (TOAEs) in the outpatient clinic of a tertiary hospital and hearing complaints of these patients. Materials and Methods: prospective cross-sectional study of TS girls aging from six to 19 years old. Results: 13 patients with TS (26 ears) were evaluated. They showed in Audiometry: normal hearing in 14 ears, NSHL in three ears, conductive in seven and mixed HL in two ears (12 alterations). In the DPOAEs: 19 absent responses ( $73.1 \%$ of cases) in at least one frequency, and in TOAEs: 16 absent responses $(61.5 \%) .15$ absent responses in both tests $(57.7 \%)$. Five patients had history of chronic otitis media. Conclusion: The presence of auditory alterations in TS patients assessed by OAE was high, with presence of history of chronic otitis media and their complications. Only one patient had hearing complaints. These findings should bring to attention HL in these patients, even without complaints.
\end{abstract}

Keywords: turner syndrome, hearing, hearing loss, otoacustic emissions

Volume 12 Issue 5 - 2020

\author{
Bettina Carvalho, Rogerio Hamerschmidt, \\ Gislaine Richter Minhoto Wiemes, Julienne \\ Angela Ramires de Carvalho, Monica Nunes \\ Lima Cat, Evaldo Dacheux de Macedo Filho \\ Otorhinolaryngology Head and Neck Surgery Department, \\ Brazil
}

Correspondence: Bettina Carvalho, Postgraduation program, HC/UFPR (Clinics Hospital of the Federal University of Parana), Otorhinolaryngology Head and Neck Surgery Department Complexo Hospital de Clínicas da UFPR. R. Gen. Carneiro, I8I - Alto da Glória, Curitiba, Brazil - PR, 80060-900, Tel +55 4l 3 360-1800, ORCID 0000-0001-7I86-4613,

Email bettinacarvalho@yahoo.com.br

Received: November II, 2020 | Published: November I8, 2020

\section{Introduction}

Turner syndrome (TS) is one of the most common genetic syndromes and affects about 1:2.000 live births. ${ }^{1}$ It was first described it in 1938 as a triad involving infantilism, webbed neck and cubitus valgus by Henry Turner first described the syndrome, and at the time otologic and auditory problems were not reported. ${ }^{2,3}$ The earliest reports of otologic diseases and their association with hearing loss (HL) in TS did not begin until the 1960's. ${ }^{4,5}$ There have been several studies on HL in TS since. However, the prevalence reported in these studies cannot be compared due to different definitions and frequencies used in the calculation of tonal average in audiometries and also the possibility that NSHL has been overestimated due to the inclusion of adults and children, and several methodology biases or confounders. ${ }^{6-8}$ The objective of this study was to evaluate the prevalence of auditory impairment in children and adolescents with Turner Syndrome, in the outpatient clinic of the Pediatric Endocrinology Unit and in the Otorhinolaryngology Department of a Tertiary University Hospital, by means of audiometry and otoacustic emissions and evaluate hearing complaints.

\section{Materials and methods}

It was an observational, cross-sectional study with prospective data collection. This study was approved by the Ethics Committee on Human Beings of the Hospital under CAAE: 34310314.8.0000.0096.

\section{Participants}

Inclusion criteria were: age between 6 and $<19$ years, with diagnosis of Turner Syndrome, part of the outpatient clinic of Pediatric Endocrinology Unit at a University Hospital, signing the consented term. There were about 24 TS patients at the outpatient clinic of the Pediatric Endocrinology Unit who come regularly for follow-ups during the period of the study. The hospital is a reference center for treatment of genetic syndromes and pediatric endocrinology and many patients come from other cities, a fact that presented some difficulty in performing new exams in this population. Before performing exams, each patient was accessed by and ENT specialist with otoscopy to rule out malformations of the ear, ear wax, or other impairments to each of the exams. If there was earwax present, the ENT specialist removed it before exams were performed.

\section{Assessment}

The exams were: Pure Tone Audiometry, Speech audiometry, Acoustic Immitance Measurements, Transient Otoacoustic Emissions (TEOAE) and Distortion Product Otoacustic Emissions (DPOAE). Patients also responded a questionnaire on otorhinolaryngologic and audiological complaints. All the auditory and electrophysiological examinations were performed by the same speech therapist at the Otorhinolaryngology department. All patients had exams performed in the same day, in order to prevent diverging results. In this study, the BETA MEDICAL ${ }^{\circledR}$ Clinical Audiometer, model Beta 6000, was used and the exams were applied in an acoustic cabin. Audiometry was used to define the type of hearing loss and threshold, threshold at $<25$ $\mathrm{dB}$ was considered normal. In TS, authors describe a type of hearing loss with a decrease in mean frequencies, but there is no consensus in the literature when considering this hearing loss. Therefore, in this study it was defined as a difference $>15 \mathrm{~dB}$ between mean frequencies and the highest and lowest frequencies. For OAE, the Capella Madsen ${ }^{\circledR}$ EOA analyzer was used. The standard equipment program for emissions recording was used. The parameters used for DPOAE were level: difference f1-f2: $10 \mathrm{~dB}, \mathrm{fl}=65, \mathrm{f} 2=55, \mathrm{Dp} 1,2$ (f1-f2), 200 sweeps, signal-to-noise ratio $>6 \mathrm{~dB}$. The frequencies evaluated are 1 , 2, 3, 4 and $6 \mathrm{kHz}$. In which $\mathrm{fl}$ is the stimulus with lower frequency and $\mathrm{f} 2$, with higher frequency. Parameters for TOAE were: non linear click, mode: fast screen, stimulus duration 40usc, polarity: 
condensation, sweeps: 2080 , noise criteria: automatic. The normality criterion considered for TOAE is $60 \%$ reproducibility and $6 \mathrm{~dB}$ signalto-noise ratio present in three or more frequency ranges. Results were analyzed qualitatively due to the sample size.

\section{Results}

Thirteen patients ( 26 ears) with TS were evaluated, aged between 6 and $<19$ years. Eight patients showed normal otoscopy at the day of the exam, two patients underwent timpanoplasty in one ear each (due to Simple chronic otitis media) and presented a neo tympanum which showed no residual perforations, one patient had a history of tympanomastoidectomy (due to Chronic suppurative otitis media) and also showed no residual perforations in the neo tympanum. One patient had a history of Chronic otitis media with effusion and use of Ventilation tubes, but otoscopy was normal at the day of the exam. The results are shown in Table 1.

Table I Results

\begin{tabular}{|c|c|c|c|c|c|c|c|c|c|}
\hline \multirow{2}{*}{ N. } & \multirow{2}{*}{ Age (Years) } & \multirow{2}{*}{ Cariotype } & \multirow{2}{*}{ TOAE } & \multicolumn{5}{|c|}{ DPOAE } & \multirow{2}{*}{ Audiometry } \\
\hline & & & & I & 2 & 3 & 4 & 6 & \\
\hline I & 6 & $45 \times, 0$ & A & A & A & A & A & A & normal \\
\hline 2 & 6 & $45 \times, 0$ & A & A & A & $A$ & A & A & normal \\
\hline 3 & 11 & $45 \times, 0$ & A & A & $P$ & $P$ & $P$ & A & normal \\
\hline 4 & 11 & $45 \times, 0$ & A & $P$ & $P$ & $P$ & $P$ & $P$ & normal \\
\hline 5 & 16 & $45 \times, 0$ & A & A & $P$ & $P$ & A & A & NSHL 6,8 kHz mild \\
\hline 6 & 16 & $45 \times, 0$ & A & A & $P$ & $P$ & $P$ & A & normal \\
\hline 7 & 15 & $45 \times, 0$ & $P$ & $P$ & $P$ & $P$ & $P$ & A & normal \\
\hline 8 & 15 & $45 \times, 0$ & $A$ & A & $P$ & $P$ & A & A & Conductive $\mathrm{HL}$ mild \\
\hline 9 & 14 & $45 \times, 0$ & A & A & A & A & A & A & Conductive HL mild \\
\hline 10 & 14 & $45 \times, 0$ & $A$ & A & $P$ & $A$ & A & A & Conductive HL mild \\
\hline II & 13 & $45 \times, 0$ & $P$ & $P$ & $P$ & $P$ & $P$ & A & normal \\
\hline 12 & 13 & $45 \times, 0$ & $P$ & $P$ & $P$ & $P$ & $P$ & A & normal \\
\hline 13 & 9 & $45 \times, 0$ & A & A & A & $P$ & $P$ & A & NSHL moderate \\
\hline 14 & 9 & $45 \times, 0$ & A & A & A & $P$ & $P$ & A & NSHL moderate \\
\hline 15 & 12 & $46 X,(x) q 10$ & A & A & A & A & A & A & Conductive $\mathrm{HL}$ moderate \\
\hline 16 & 12 & $46 X,(x) q 10$ & A & $P$ & $P$ & $P$ & A & A & Conductive $\mathrm{HL}$ mild $500 \mathrm{~Hz}$ \\
\hline 17 & 15 & $45 X / 46 X X$ & $P$ & $P$ & $P$ & A & $P$ & A & normal \\
\hline 18 & 15 & $45 X / 46 X X$ & $P$ & $P$ & $P$ & $P$ & $P$ & $P$ & normal \\
\hline 19 & 9 & $45 \times, 0$ & $P$ & $P$ & $P$ & $P$ & $P$ & $P$ & Conductive $\mathrm{HL}$ mild \\
\hline 20 & 9 & $45 \times, 0$ & $P$ & $P$ & $P$ & $P$ & $P$ & $P$ & Conductive HL mild \\
\hline 21 & 16 & $45 \times, 0$ & $P$ & $P$ & $P$ & $P$ & $P$ & $P$ & normal \\
\hline 22 & 16 & $45 \times, 0$ & A & $P$ & $P$ & $P$ & $P$ & A & normal \\
\hline 23 & 19 & $45 X / 46 X X$ & $P$ & $P$ & $P$ & $\mathrm{P}$ & $P$ & $P$ & normal \\
\hline 24 & 19 & $45 X / 46 X X$ & $P$ & $P$ & $P$ & $P$ & $P$ & $P$ & normal \\
\hline 25 & 18 & $45 \times, 0$ & A & A & A & A & A & A & Mixed HL moderate \\
\hline 26 & 18 & $45 \times, 0$ & A & $A$ & $A$ & A & A & A & Mixed HL moderate \\
\hline
\end{tabular}

Note:TOAE (Transient Otoacustic Emissions), DPOAE (Distortion product Otoacustic Emissions), A (Absent), P (Present) for frequency in KHz.

As for Audiometry, TS patients presented normal results in 14 ears $(53.8 \%)$ and altered in 12 ears $(46.2 \%)$, with conductive hearing loss in seven, NSHL in three, and mixed in two. As for the degree of hearing loss: mild in seven ears (six conductive and one NS), and moderate in five ears (two mixed, one conductive, two NS). We did not observe any patient with severe or profound hearing loss. Impedances were altered in four ears (curve type $\mathrm{C}$ and $\mathrm{B}$ ), the others were within normal (curve type A or As). Some patients had present OAE despite having a conductive hearing loss, which was due to conductive hearing loss being present in lower frequencies while OAE evaluated frequencies higher than $1 \mathrm{kHz}$. Other patients had absent OAE despite having a normal audiometry, which we believe could be due to disfunction of the auditory tube (common in children) but could also indicate early neurosensorial hearing loss. Even though some frequencies of DPOAE may be absent in certain patients the concomitant absence of TOAE tend to confirm the alteration in the exam, reason why we normally perform both tests in our patients. One patient had complaints of Rhinitis, which was under treatment. Only the patient who underwent tympanomastoidectomy complained of a hearing impairment. No patients had severe craniofacial malformations in our study. No new imaging studies were performed in these patients. 


\section{Discussion}

Auditory assessment includes subjective and objective audiological tests. Subjective tests are less accurate because they depend on the patient's response, which may interfere with the outcome, and it may be influenced by the child's interest, cognition, and participation, which require skills, experience, and patience from the examiner. These tests include pure tone and speech audiometry. Objective audiological tests are more precise and include acoustic immitance measurement, evaluation of Otoacoustic Emissions (OAE) and Auditory Evoked Potentials (Such as Brainstem Evoked Auditory Response - BERA).

OAE primarily seek to evaluate if the cochlea presents normal function. The examination is painless, noninvasive, fast, has low cost and high sensitivity. A normal result indicates integrity of cochlear physiology for the normal social hearing level, which is up to $25 \mathrm{~dB}$ so they can be used for hearing screening. But results may suffer by the condition of the middle ear. ${ }^{9}$ Mechanisms involved in HL in TS are thought to be recurrent otitis media and dysfunction of the Eustachian tube, leading to conductive HL, and many genetic mechanisms such as cell cycle delay hypothesis, IGF-1 deficiency and estrogen deficiency are thought to play a role in NSHL. ${ }^{10,11}$ In studies based on audiometry the percentage of $\mathrm{HL}$ on TS patients raged between 0 to $47 \%$ for conductive, 3 to $28 \%$ for mixed and 10 to $82 \%$ for NSHL in medium frequencies and 9 to $83 \%$ for NSHL in high frequencies. These percentages are highly variable due to the problems with respect to methodology (retrospective, questionnaire-based studies, recruitment bias, different definitions of HL, loss of patients in the follow-up and confusion in the results due to the inclusion of mixed HL without separation between conductive or neurosensory components, and without the indication of contribution of chronic otitis media to levels of bone conduction) of the existing studies. ${ }^{8}$ Most recent studies, based on objective tests (such as OAE), aim to standardize the information regarding $\mathrm{HL}$ in TS. ${ }^{6,12-14}$

Serra et al. [12] evaluated 21 patients with a mean age of 15 years through TOAE. They observed absence of response in $71.4 \%$ (15 patients, 28 ears). They also found predominance of conductive hearing loss $(42.8 \%)$ with evidence of chronic otitis media. Morimoto et al. [13] evaluated 33 patients, aged between 8 and 40 years, through TOAE and DPOAE. TOAEs were absent in 16 cases ( 28 ears - 42.4\%) and DPOAEs in 20 cases ( 31 ears - 47.0\%). The authors did not specify in which frequencies the DPOAEs were absent, only reported that the missing EOA were in the cases with PANS in acute frequencies and in the conductive HL. The audiometric alterations were present in $51.5 \%$ of the cases. We found in 46,15\% (12 ears). Gawron et al. [14] evaluated 51 patients with a mean age of 14.3 years. The audiometric findings were altered in $36.3 \%$ of the ears, while the DPOAE alterations were present in $41.4 \%$ of the ears, reflecting HL with a cochlear location that was not clinically apparent. They described OAE results as almost completely matching audiometry findings.

Hederstierna et al. [6] evaluated 30 patients with mean age of 52.2 years using TOAE, Audiometry and BERA and their findings suggest a cochlear origin of NSHL that commonly affects TS. The authors sought to evaluate only ST patients who reportedly would not have benefited from estrogen replacement at puberty, therefore considering that estrogen replacement affects hearing, perhaps these results would be different in a population that required estrogen replacement. They also chose only patients with mild to moderate HL, thus not representing the entire population of TS patients. The present study aimed only to evaluate children and adolescents with TS, thus eliminating the possibility of early presbicusis in these patients.
Our results correspond to alterations that are already present since childhood and adolescence (even with normal audiometry results in some patients). Some patients had alterations due to chronic otitis media, which appeared common for these patients $(38.5 \%$ of the patients (5/13)). Considering complaints reported by the patients or those responsible in the questionnaires, only one presented hearing complaints. It suggests that one cannot rely only on the subjective complaints of these patients, and routine screening should be performed with a thorough otorhinolaryngology evaluation.

In this case it is difficult to compare results with the literature since previous studies did not evaluate both TOAE and DPOAE, or the same age group. What we also wanted to show is how hearing evaluation should not be done with single exams, but with a complete evaluation when possible. It appears that karyotype is important in the HL in TS, which is increased in patients with either monossomy 45,X or isochromossome 46,I (Xq). Most of our patients fell into these categories, which may have contributed to our results. ${ }^{11}$ Even though some may consider not useful to perform OAE in conductive or mixed hearing loss, we believe bringing attention to these patients by the pediatric community is important. Previous studies also addressed patients with these types of hearing losses with OEA. ${ }^{13}$ But the most important is that otorhinolaryngologists have this in mind. Even as some authors may consider each ear as the mirror to the other, we preferred to view results individually per ear, as it was done in previous articles. In the future, more studies focused on the evaluation of HL present in OAE prior to audiometry and in younger patients should be recommended, investigating possible causes of hearing loss but most importantly seeking its prevention.

\section{Conclusion}

The presence of auditory impairment in TS patients assessed by OAE was high in this population. They were higher than those found in audiometry. There was also a great prevalence of chronic otitis media history in these patients which can be a confounder for the OAE. Complaints of subjective hearing loss were not so frequent, showing the need for routine hearing evaluation of these patients.

\section{Acknowledgment}

None.

\section{Conflicts of interest}

The author declares that there is no conflict of interest to disclose.

\section{Funding}

None.

\section{References}

1. Gravholt $\mathrm{CH}$. Epidemiological, endocrine and metabolic features in Turner syndrome. Eur J Endocrinol. 2004;151(6):657-687.

2. Turner HH. A syndrome of infantilism, congenital webbed neck, and cubitus valgus. Endocrinology. 1938;23(5):566-574.

3. Hultcrantz M, Stenberg AE, Fransson A, et al. Characterization of hearing in an X,0 'Turner mouse'. Hearing Research. 2000;143(12):182-188.

4. Szpunar J, Rybak M. Middle ear disease in Turner's syndrome. Arch Otolaryngol. 1968;87(1):34-40.

5. Anderson H, Filipsson R, Fluur E, et al. Hearing impairment in Turner's syndrome. Acta Otolaryngol. 1969:Suppl 247:1-26. 
6. Hederstierna C, Hultcrantz M, Rosenhall U. Estrogen and hearing from a clinical point of view; characteristics of auditory function in women with Turner syndrome. Hear Res. 2009;252(1-2):3-8.

7. Parkin M, Walker P. Hearing loss in Turner syndrome. Int J Pediatr otorhinolaryngol. 2009;73(2):243-247.

8. Dhooge IJM, De Vel E, Verhoye C, et al. Otologic disease in Turner Syndrome. Otol Neurotol. 2005;26(2):145-150.

9. Vieira ABC, Macedo LR, Goncalces DU. O diagnóstico da perda auditiva na infância. Pediatria. 2007;29(1):43-49.

10. Alves A, Oliveira CS. Hearing loss among patients with Turner's sydrome: literature review. Braz J Otorhinolaryngol. 2014;80(3):257263.
11. Bonnard A, Bark R, Hederstierna C. Clinical update on sensorineural hearing loss in Turner syndrome and the $\mathrm{X}$-Chromossome. Am J Med Genet. 2019;181(1):1-7.

12. Serra A, Cocuzza S, Caruso E, et al. Audiological range in Turner's syndrome. Int J Pediatr otorhinolaryngol. 2003;67(8):841-845.

13. Morimoto N, Tanaka T, Taiji H, et al. Hearing loss in Turner Syndrome. J Pediatr. 2006;149(5):697-701.

14. Gawron W, Wikiera B, Rostkowska- Nadolska B, et al. Evaluation of hearing organ in patients with Turner syndrome. Int $J$ Pediatr Otorhinolaryngol. 2008;72(5):575-579. 